\title{
The prognostic value of pulmonary embolism severity index in acute pulmonary embolism: a meta-analysis
}

\author{
Xiao-Yu Zhou', Su-Qin Ben ${ }^{1}$, Hong-Lin Chen ${ }^{2}$ and Song-Shi Ni ${ }^{1 *}$
}

\begin{abstract}
Background: Prognostic assessment is important for the management of patients with acute pulmonary embolism (APE). Pulmonary Embolism Severity Index (PESI) and simple PESI (sPESI) are new emerged prognostic assessment tools for APE. The aim of this meta-analysis is to assess the accuracy of the PESI and the sPESI to predict prognostic outcomes (all-cause and PE-related mortality, serious adverse events) in APE patients, and compare between these two PESIs.
\end{abstract}

Methods: MEDLINE and EMBASE database were searched up to June 2012 using the terms "Pulmonary Embolism Severity Index" and "pulmonary embolism". Summary odds ratio (OR) with 95\% confidence intervals (Cls) for prognostic outcomes in low risk PESI versus high risk PESI were calculated. Summary receiver operating characteristic curve (SROC) used to estimate overall predicting accuracies of prognostic outcomes.

Results: Twenty-one studies were included in this meta-analysis. The results showed low-risk PESI was significantly associated with lower all-cause mortality (OR $0.13 ; 95 \% \mathrm{Cl} 0.12$ to 0.15$)$, PE-related mortality (OR 0.09; $95 \% \mathrm{Cl} 0.05$ to 0.17 ) and serious adverse events (OR $0.34 ; 95 \% \mathrm{Cl} 0.29$ to 0.41 ), with no homogeneity across studies. In sPESI subgroup, the OR of all-cause mortality, PE-related mortality, and serious adverse events was $0.10(95 \% \mathrm{Cl} 0.08$ to 0.14), 0.09 (95\% Cl 0.03 to 0.26 ) and 0.40 (95\% Cl 0.31 to 0.51 ), respectively; while in PESI subgroup, the OR was 0.14 ( $95 \% \mathrm{Cl} 0.13$ to 0.16$), 0.09$ (95\% Cl 0.04 to 0.21 ), and 0.30 (95\% Cl 0.23 to 0.38 ), respectively. For accuracy analysis, the pooled sensitivity, the pooled specificity, and the overall weighted AUC for PESI predicting all-cause mortality was 0.909 ( $95 \%$ Cl: 0.900 to 0.916), 0.411 ( $95 \%$ Cl: 0.407 to 0.415 ), and 0.7853 \pm 0.0058 , respectively; for PE-related

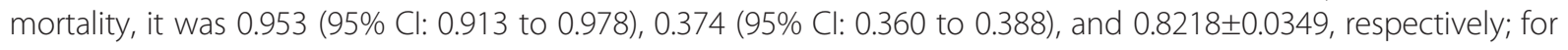
serious adverse events, it was 0.821 ( $95 \%$ Cl: 0.795 to 0.845), 0.389 (95\% Cl: 0.384 to 0.394), and 0.6809 \pm 0.0208 , respectively. In SPESI subgroup, the AUC for predicting all-cause mortality, PE-related mortality, and serious adverse events was $0.7920 \pm 0.0117,0.8317 \pm 0.0547$, and $0.6454 \pm 0.0197$, respectively. In PESI subgroup, the AUC was 0.7856 $\pm 0.0075,0.8158 \pm 0.0451$, and $0.6609 \pm 0.0252$, respectively.

Conclusions: PESI has discriminative power to predict the short-term death and adverse outcome events in patients with acute pulmonary embolism, the PESI and the sPESI have similar accuracy, while sPESI is easier to use. However, the calibration for predicting prognosis can't be calculated from this meta-analysis, some prospective studies for accessing PESI predicting calibration can be recommended.

Keywords: Acute pulmonary embolism, Pulmonary embolism severity index, Prognosis

\footnotetext{
* Correspondence: pphss@126.com

'Department of Respiratory Diseases, the Affiliated Hospital of Nantong University, Xi Si Road 20\#, Nantong City, Jiangsu Province 226001, People's Republic of China

Full list of author information is available at the end of the article
}

\section{Biomed Central}

(c) 2012 Zhou et al.; licensee BioMed Central Ltd. This is an Open Access article distributed under the terms of the Creative Commons Attribution License (http://creativecommons.org/licenses/by/2.0), which permits unrestricted use, distribution, and reproduction in any medium, provided the original work is properly cited. 


\section{Background}

Epidemiological studies showed the prevalence of acute pulmonary embolism (APE) among hospitalized patients in the United States, according to data collected between 1979 and 1999, was 0.4\% [1]. In Europe, a total of 67,351 (in 2005), 69,234 (in 2006) and 71,223(in 2007) APE were coded either as principal or secondary diagnosis in German hospitals according to the data from Federal Statistical Office [2]. In Asia, from a multi-center registration study of Chinese hospitals between 1997 and 2008, a total of 18,206 patients were confirmed with APE from $16,972,182$ hospital admissions, the annual incidence was $0.1 \%$ (95\% CI: $0.1 \%$ to $0.2 \%$ ) [3]; while in Korean, incidence of established APE was $88(0.17 \%)$ of 50,882 identified retrospectively from patients hospitalized during a 2-year period from 2005 to 2007[4]. APE has become a relatively common cardiovascular emergency.

The key consequences of a pulmonary thromboembolic episode are haemodynamic. Large and multiple emboli might abruptly increase pulmonary vascular resistance to a level of after load which cannot be matched by the right ventricle (RV), and sudden death may occur [5]. In the ICOPER registry, the 14-day mortality of APE was above $20 \%$ [6]. In the RIETE registry, at 3 months, the cumulative rates of overall mortality and fatal PE were $8.65 \%$ and $1.68 \%$, respectively [7]. While in the EMPEROR registry, the all-cause 30-day mortality rate was $5.4 \%$ (95\% CI: $4.4 \%$ to $6.6 \%$ ) [8]. APE has become one of the leading causes of preventable hospital deaths.

The mortality of APE can be predicted by haemodynamic status, right ventricular dysfunction, myocardial injury, and other clinical and routine laboratory tests. Shock and hypotension are principal markers of high risk of early death in APE. RV dysfunction which is detected by echocardiography, computed tomography, and brain natriuretic peptide (BNP) is related to intermediate risk of short-term mortality in APE. Myocardial injury in patients with APE is related to an intermediate risk of short-term mortality, which can be detected by troponin $\mathrm{T}$ or I testing [5]. While the Pulmonary Embolism Severity Index (PESI) was recently found to be a well validated and highly reliable clinical prognostic model for patients with APE.

The PESI was designed by Aujesky D and his colleague in 2005, which comprises 11 routinely available clinical predictor variables with different prognostic weights [9]. On the basis of the PESI score, each patient is classified into one of five classes (I-V), with 30day mortality ranging from $1.1 \%$ to $24.5 \%$. Patients in risk classes I and II are categorized as low-risk and in risk classes III-V are categorized as high-risk. While the PESI is hard to memorizes for based on 11 items, and it's difficult to calculate in busy emergency departments. The simplified PESI (sPESI) was developed in 2010, which includes six of the 11 original PESI variables [10]. Patients with none of the variables (0 points) are categorized as low-risk; with one to six the variables (1-6 points) are categorized as high-risk. The PESI and sPESI are listed in the Additional file 1: Appendix 1.

The prognostic value of the PESI and sPESI has been assessed by some studies, but has not been systematic reviewed. We conduct this meta-analysis to assess the accuracy of the PESI and the sPESI to predict prognostic outcomes in APE patients. In particular, we also compared the predicting accuracy between these two PESIs.

\section{Materials and methods Eligibility criteria}

We defined studies as being eligible for inclusion in this analysis if they met the following criteria: (1) types of studies: observational studies ( cohort studies and casecontrol studies ) were included; (2) types of participants: population of objective diagnosis of APE with shortterm prognosis outcome were included; (3) types of outcomes: studies included at least one of three prognostic outcomes: all-cause mortality, PE-related mortality, serious adverse events; and a $2 \times 2$ table of outcome results could be constructed based on low-risk PESIs or highrisk PESIs.

\section{Search strategy and study selection}

We searched MEDLINE (PubMed, http://www.ncbi.nlm. nih.gov/pubmed/), and EMBASE (http://www.embase. com) up to June 2012. The search strategy included terms of "Pulmonary Embolism Severity Index" and "Pulmonary Embolism". The search was limited to English- language articles. We did not appoint limit in country, race, or publication year. To identify any additional relevant studies, we also hand-searched conference proceedings and scanned references of retrieved articles.

Study selection was initially performed by review of titles and abstracts. When there was any possibility that it might be relevant, the full text were downloaded and then reviewed for data retrieval.

Two reviewers independently judged study eligibility while screening the citations. Disagreements were resolved by a third reviewer.

\section{Quality assessment}

We used Newcastle-Ottawa Scale (NOS) to assess the quality of the included observational studies [11]. The NOS contains eight items, categorized into three dimensions including Selection (4), Comparability (1), and Exposure (3). A high quality study can be awarded a maximum of one star for each numbered item within the Selection and Exposure categories. A maximum of two stars can be given for Comparability. The NOS 
ranges between zero up to nine stars. The NOS is listed in the Additional file 2: Appendix 2.

Two reviewers independent assessed the quality of studies. Disagreements were resolved by a third reviewer.

\section{Data extraction}

We collected information about study characteristics (study year, country, study design, number of patients, mean age, gender distribution, type of PESI, methods for diagnosis of APE, haemodynamic status, length of follow-up) and number of patients with the available outcomes of all-cause death, PE-related death, serious adverse events among low-risk or high-risk PESIs.

In this meta-analysis, all-cause mortality was considered as the primary outcome, PE-related death, serious adverse events were considered as the secondary outcome. Serious adverse events were defined as any of the following: nonfatal recurrent PE, nonfatal recurrent DVT, nonfatal bleeding, or delayed homodynamic instability.

Data were extracted independently by two reviewers. Any differences of opinion were resolved by discussion and consensus reached by discussion with a third reviewer.

\section{Statistical analysis}

First, we considered high-risk PESI as a risk factor for APE prognostic adverse outcomes. Summary OR with 95\% CIs for three prognostic outcomes in low-risk PESI versus high-risk PESI was calculated respectively. Statistical heterogeneity was explored by $x^{2}$ and inconsistency $\left(\mathrm{I}^{2}\right)$ statistics; an $\mathrm{I}^{2}$ value of 50 percent or more represented heterogeneity [12]. If there was no heterogeneity, fixed effects model was used for meta-analysis; otherwise, a random effect model based on the DerSimonian and Laird estimator was used [13]. Overall effects were determined using the $\mathrm{Z}$ test. Potential publication bias was evaluated by the funnel plot. Subgroup meta-analyses were performed by PESI subgroup and sPESI subgroup. Sensitivity analysis was performed by only pooled prospective studies. The first step meta-analysis was performed with Review Manager (RevMan) software (version 5.0.21; Update Software Ltd, Oxford, Oxon, UK).

Second, we assess the accuracy (discriminative power) of PESI to predict APE prognostic outcomes. If we predict low-risk PESI as good prognostic outcomes, highrisk PESI as worse prognostic outcomes, the sensitivity (true positive rate) and specificity (true negative rate) of each included study was calculated compared to actual prognostic outcomes (the so-called gold standard). And the overall pooled sensitivity and specificity with 95\% CIs was estimated by DerSimonian and Laird's randomeffects model [13]. In addition, summary receiver operator characteristic (SROC) analysis was performed to examine the interaction between sensitivity and (1-specificity) [14], and to quantify test performance using the area under the curve (AUC) and $\mathrm{Q}^{*}$ value[15]. The second step metaanalyses were performed with Meta DiSc 1.4 (version 0.6; By Joseph Lau) [16].

\section{Results}

Description of included studies

Overall, 21 studies [10,17-36] were selected for this meta-analysis described in Figure 1. Among those studies, 10 studies were prospective cohorts, 9 studies were retrospective cohorts or case-control studies, the remaining 2 studies didn't mention about the study design type. Eleven studies were multi-center studies. Countries involved in these studies were Germany, Spain, Poland, Greece, Switzerland, USA, Belgium, Italy, Greece, UK, Israel, and Korea. Most of the studies (20 studies) came from Europe and North America, only a study came form Asia. All of the 21 included studies used the same diagnostic criteria of $\mathrm{PE}$, which included high clinical probability of $\mathrm{PE}$, and confirmed by contrast-enhanced multi detector $\mathrm{CT}$ or ventilationperfusion lung scan, or confirmed lower limb deep vein thrombosis (DVT) by venous ultrasound. A total of 5 studies included haemodynamic stable patients, a study included haemodynamic stable and unstable patients, but most of the studies didn't mention about the haemodynamic status of the included patients. The length of follow-up ranged from hospital discharge to 6 month following admission to hospital. The NOS for methodological quality assessment of these 21 studies all scored at 7 of 8 star, indicating good quality. The main characteristics of the selected studies are reported in Table 1.

\section{Meta-analysis}

High-risk PESI as a risk factor for adverse outcomes

The association between PESI and PE adverse prognosis outcome risk of every included study are shown in Table 2. Data on all-cause mortality were reported in 19 studies. Five studies assessed the SPESI, 11 studies assessed the PESI, 3 studies assessed sPESI and PESI in the same study. A total of 50,021 patients were analyzed in the studies, including 4,991 patients with allcause death. There was no heterogeneity in the studies $\left(\mathrm{I}^{2}=0 \%\right)$. The pooled all-cause mortality was $2.0 \%$ (456/22978) in patients with low-risk PESI Vs $16.7 \%$ (4535/27043) in patients with high-risk PESI. The summary OR of patients with low-risk PESI compared with patients with high-risk PESI was 0.13 (95\% CI 0.12 to $0.15 ; \mathrm{Z}=33.76, \mathrm{P}<0.00001$ ) (Figure 2). The funnel plot showed no asymmetry, which indicated no evidence of publication bias (Figure 3A). While in subgroup analysis for SPESI and PESI, the pooled all-cause mortality was $1.8 \%(180 / 9995)$ in patients with low-risk sPESI Vs $25.2 \%(2040 / 8096)$ in patients with high-risk sPESI, the summary OR of patients with low-risk sPESI compared 
with patients with high-risk sPESI was 0.10 (95\% CI 0.08 to $0.14 ; \mathrm{Z}=15.04, \mathrm{P}<0.00001$ ) (Figure 2 Above); the pooled all-cause mortality was $2.1 \%(276 / 12983)$ in patients with low-risk PESI Vs 13.2\% (2495/18947) in patients with high-risk PESI, the summary OR of patients with low-risk PESI compared with patients with high-risk PESI was 0.14 (95\% CI 0.13 to 0.16 ; $\mathrm{Z}=30.10, \mathrm{P}<0.00001$ ) (Figure 2 Below).

Data on PE-related mortality were reported in 7 studies, 3 studies assessed the sPESI, and 4 studies assessed the PESI. A total of 4,794 patients were analyzed in the studies, including 192 patients with PE-related death. There was no heterogeneity in the studies $\left(\mathrm{I}^{2}=0 \%\right)$. The pooled PE-related mortality was $0.5 \%$ (9/1731) in patients with low-risk PESI Vs 6.0\% (183/3063) in patients with high-risk PESI. The summary OR of patients with low-risk PESI compared with patients with high-risk PESI was 0.09 (95\% CI 0.05 to 0.17 ; $Z=7.14$, $\mathrm{P}<0.00001$ ) (Figure 4). The funnel plot showed no asymmetry, which indicated no evidence of publication bias (Figure 3B). While in subgroup analysis for sPESI and PESI, the pooled PE-related mortality was $0.4 \%$ (3/770) in patients with low-risk sPESI Vs 5.1\% (72/1404) in patients with high-risk sPESI, the summary OR of patients with low-risk sPESI compared with patients with high-risk sPESI was 0.09 (95\% CI 0.03 to 0.26 ; $\mathrm{Z}=4.39, \mathrm{P}<0.00001$ ) (Figure 4 Above); the pooled PErelated mortality was $0.6 \%(6 / 961)$ in patients with low-risk PESI Vs 6.7\% (111/1659) in patients with high-risk PESI, the summary OR of patients with lowrisk PESI compared with patients with high-risk PESI was 0.09 (95\% CI 0.04 to $0.21 ; Z=5.62, P<0.00001$ ) (Figure 4 Below).

Data on serious adverse events were reported in 8 studies, 4 studies assessed the sPESI, 3 studies assessed the PESI, a study assessed sPESI and PESI in the same study. A total of 50,021 patients were analyzed in the studies, including 4,991 patients with serious adverse events. There was no heterogeneity in the studies $\left(\mathrm{I}^{2}=36 \%\right)$. The pooled serious adverse events rate was $1.2 \%(166 / 13355)$ in patients with low-risk PESI Vs $3.5 \%$ $(761 / 21621)$ in patients with high-risk PESI. The summary OR of patients with low-risk PESI compared with patients with high-risk PESI was 0.34 (95\% CI 0.29 to 0.41; $\mathrm{Z}=12.32, \mathrm{P}<0.00001$ ) (Figure 5). The funnel plot showed no asymmetry, which indicated no evidence of publication bias (Figure 3C). While in subgroup analysis for sPESI and PESI, the pooled serious adverse events 
Table 1 Characteristics of Selected Studies

\begin{tabular}{|c|c|c|c|c|c|c|c|c|}
\hline First author, Year & Country & Study design & $\begin{array}{l}\text { Patients } \\
\text { (n) }\end{array}$ & $\begin{array}{l}\text { Age } \\
\text { (Year) }\end{array}$ & $\begin{array}{l}\text { Gender } \\
\text { (male/female) }\end{array}$ & Haemodynamic & Follow-Up & $\begin{array}{l}\text { NOS } \\
(*)\end{array}$ \\
\hline Lankeit M, 2011 & $\begin{array}{l}\text { Germany, Spain, } \\
\text { Poland, Greece }\end{array}$ & Prospective & 526 & $71(55-79)$ & $266 / 260$ & Stable & 6 month & 8 \\
\hline Sánchez D, 2011 & Spain, Switzerland, USA & Retrospective & 1291 & $74(61-80)$ & $579 / 712$ & Stable and unstable & 30 day & 7 \\
\hline Lankeit M,2012 & Germany, Spain, Greece & Prospective & 526 & $74(61-80)$ & $227 / 299$ & Stable and unstable & 30 day & 7 \\
\hline Righini M,2011 & $\begin{array}{l}\text { Switzerland, France, } \\
\text { Belgium }\end{array}$ & Prospective & 357 & mean age 64 & $157 / 200$ & Stable and unstable & 3 month & 8 \\
\hline Spirk D, 2011 & Switzerland & Prospective & 369 & $20.9 \%>80$ & 195/174 & Stable and unstable & 30 day & 8 \\
\hline Vanni S, 2011 & Italy & Prospective & 463 & $67 \%>65$ & $208 / 254$ & Stable and unstable & in-hospital stay & 8 \\
\hline Venetz C, 2011 & Switzerland, Spain & Not mentioned & 15531 & $67(52-77)$ & $6227 / 9304$ & Stable and unstable & 30 day & 7 \\
\hline Hariharan P, 2011 & USA & Retrospective & 245 & $57 \pm 17$ & $115 / 130$ & Stable and unstable & in-hospital stay & 8 \\
\hline Jiménez D, 2011 & $\begin{array}{l}\text { Spain, Switzerland, } \\
\text { USA, Greece }\end{array}$ & Not mentioned & 591 & $74(65-82)$ & $254 / 337$ & Stable & 30 day & 7 \\
\hline $\begin{array}{l}\text { Singanayagam } \\
\mathrm{A}, 2011\end{array}$ & UK & Retrospective & 411 & $55.4 \%>65$ & $177 / 234$ & Stable and unstable & 30 day & 8 \\
\hline $\begin{array}{l}\text { Jiménez D, } 2010 \\
\text { (Derivation Cohort) }\end{array}$ & $\begin{array}{l}\text { Spanish, France, } \\
\text { Italy, Israel }\end{array}$ & Retrospective & 10354 & $52.8 \%>65$ & $4100 / 6254$ & Stable and unstable & 30 day & 7 \\
\hline $\begin{array}{l}\text { Jiménez D, } 2010 \\
\text { (Derivation Cohort) }\end{array}$ & $\begin{array}{l}\text { Spanish, France, } \\
\text { Italy, Israel }\end{array}$ & Retrospective & 995 & $67.4 \%>65$ & $449 / 546$ & Stable and unstable & 30 day & 7 \\
\hline $\begin{array}{l}\text { Jiménez D, } 2010 \\
\text { (RIETE Cohort) }\end{array}$ & $\begin{array}{l}\text { Spanish, France, } \\
\text { Italy, Israel }\end{array}$ & Retrospective & 7106 & $68.2 \%>65$ & $3232 / 3874$ & Stable and unstable & 30 day & 7 \\
\hline Sam A, 2011 & $\begin{array}{l}\text { Spain, USA, } \\
\text { Switzerland }\end{array}$ & Prospective & 1206 & $69.3 \pm 16.1$ & $536 / 670$ & Stable and unstable & 30 day & 8 \\
\hline Chan CM, 2010 & USA & Retrospective & 302 & $59.7 \pm 17.2$ & 133/169 & Stable and unstable & 90 day & 8 \\
\hline $\begin{array}{l}\text { Singanayagam A, } \\
2010\end{array}$ & UK & Retrospective & 585 & $55.4 \%>65$ & $258 / 327$ & Stable and unstable & 30 day & 7 \\
\hline Moores L, 2010 & $\begin{array}{l}\text { USA, Switzerland, } \\
\text { Spain }\end{array}$ & Retrospective & 567 & $74 \%>65$ & $245 / 322$ & Stable & 30 day & 7 \\
\hline Nordenholz K, 2011 & USA & Retrospective & 168 & $53(40-66)$ & $72 / 96$ & Stable and unstable & 30 day & 7 \\
\hline Choi WH,2009 & Korea & Retrospective & 90 & $60.4 \pm 16.0$ & $37 / 53$ & Stable and unstable & 30 day & 7 \\
\hline Donzé J, 2008 & Switzerland, France, USA & Prospective & 357 & $64 \pm 18$ & 158/199 & Stable & 90 day & 8 \\
\hline Jiménez D, 2008 & Spain, USA & Prospective & 318 & $75.5 \%>65$ & $136 / 182$ & Stable & 30 day & 8 \\
\hline $\begin{array}{l}\text { Jiménez D, } 2007 \\
\text { (Validation Cohort) }\end{array}$ & Spain & Prospective & 599 & $67 \%>65$ & $246 / 353$ & $\begin{array}{l}\text { Stable and } \\
\text { unstable }\end{array}$ & 30 day & 8 \\
\hline Aujesky D,2007 & Switzerland, France, USA & Prospective & 899 & $64 \%>65$ & $382 / 517$ & Stable and unstable & 30 day & 8 \\
\hline
\end{tabular}

rate was $1.3 \%(85 / 6553)$ in patients with low-risk sPESI Vs $2.9 \%(377 / 11605)$ in patients with high-risk sPESI, the summary OR of patients with low-risk sPESI compared with patients with high-risk sPESI was 0.40 (95\% CI 0.31 to $0.51 ; Z=7.59, \mathrm{P}<0.00001$ ) (Figure 5 Above); the pooled serious adverse events rate was $1.2 \%(81 /$ 6802) in patients with low-risk PESI Vs 3.8\% (384/ 10016 ) in patients with high-risk PESI, the summary OR of patients with low-risk PESI compared with patients with high-risk PESI was 0.30 (95\% CI 0.23 to 0.38 ; $\mathrm{Z}=9.81, \mathrm{P}<0.00001$ ) (Figure 5 Below).

Sensitivity analysis of primary outcome (all-cause mortality) by removal the retrospective studies showed there was no heterogeneity in the studies $\left(\mathrm{I}^{2}=1 \%\right)$; the summary OR of patients with low-risk PESI compared with patients with high-risk PESI was 0.13 (95\% CI 0.10 to 0.19; $\mathrm{Z}=11.81, \mathrm{P}<0.00001$ ) (Figure 6); the funnel plot showed no asymmetry, which indicated no evidence of publication bias (Figure 3D). Sensitivity analyses showed the robust result of the pooled meta-analyses.

\section{Accuracy of the PESI to predict prognostic outcomes}

The sensitivity and specificity for PESI in PE prognosis diagnosis of each included studies are also shown in Table 2.

For the outcome of all-cause mortality, the pooled sensitivity was 0.909 (95\% CI: 0.900 to 0.916$)$. The pooled specificity was 0.411 (95\% CI: 0.407 to 0.415 ). The SROC curve is shown in Figure 7A. The overall weighted AUC 
Table 2 PESI for PE risk and diagnosis

\begin{tabular}{|c|c|c|c|c|c|c|c|c|c|c|c|c|c|}
\hline \multirow{3}{*}{$\begin{array}{l}\text { First author, } \\
\text { Year }\end{array}$} & \multirow[t]{3}{*}{ PESI } & \multicolumn{6}{|c|}{ Risk } & \multicolumn{6}{|c|}{ Diagnosis } \\
\hline & & \multicolumn{2}{|c|}{ All-cause death } & \multicolumn{2}{|c|}{ PE-related death } & \multicolumn{2}{|c|}{ Adverse outcome } & \multicolumn{2}{|c|}{ All-cause death } & \multicolumn{2}{|c|}{ PE-related death } & \multicolumn{2}{|c|}{ Adverse outcome } \\
\hline & & PESI low risk & PESI high risk & PESI low risk & PESI high risk & PESI low risk & PESI high risk & Sensitivity & Specific & Sensitivity & Specific & Sensitivity & Specific \\
\hline Lankeit M, 2011 & sPESI & $1 / 198(0.5 \%)$ & $26 / 328(7.9 \%)$ & 0/198 (0.0\%) & $8 / 328(2.4 \%)$ & $2 / 198(1.0 \%)$ & $29 / 328(8.8 \%)$ & 0.963 & 0.395 & 1.000 & 0.382 & 0.935 & 0.396 \\
\hline Sánchez D, 2011 & sPESI & $7 / 407$ (1.7\%) & 120/884 (13.6\%) & $2 / 407(0.5 \%)$ & $58 / 884(6.6 \%)$ & Not mentioned & Not mentioned & 0.945 & 0.344 & 0.967 & 0.329 & N/A & N/A \\
\hline Lankeit M, 2012 & sPESI & 0/165 (0.0\%) & 40/361 (11.1\%) & Not mentioned & Not mentioned & $3 / 165(1.8 \%)$ & $18 / 361(5.0 \%)$ & 1.000 & 0.340 & N/A & N/A & 0.857 & 0.321 \\
\hline Righini M, 2011 & sPESI & $1 / 165(0.9 \%)$ & 10/192 (5.2\%) & 1/165 (0.9\%) & 6/192 (3.0\%) & Not mentioned & Not mentioned & 0.909 & 0.474 & 0.857 & 0.469 & N/A & N/A \\
\hline Spirk D, 2011 & sPESI & 0/106 (0.0\%) & $16 / 263(6.1 \%)$ & Not mentioned & Not mentioned & $3 / 106(2.9 \%)$ & $21 / 263(8.0 \%)$ & 1.000 & 0.394 & N/A & N/A & 0.875 & 0.299 \\
\hline Vanni S, 2011 & PESI & $4 / 145(2.8 \%)$ & $34 / 318(10.7 \%)$ & $1 / 145(0.7 \%)$ & $24 / 318(7.5 \%)$ & $3 / 145$ (2.1\%) & $29 / 318$ (9.1\%) & 0.895 & 0.332 & 0.960 & 0.329 & 0.906 & 0.329 \\
\hline Venetz C, 2011 & PESI & $\begin{array}{l}146 / 6332 \\
(2.3 \%)\end{array}$ & $\begin{array}{l}1234 / 9179 \\
(14.1 \%)\end{array}$ & Not mentioned & Not mentioned & $\begin{array}{l}63 / 6332 \\
(1.0 \%)\end{array}$ & $\begin{array}{l}275 / 9179 \\
(3.0 \%)\end{array}$ & 0.894 & 0.438 & N/A & N/A & 0.814 & 0.417 \\
\hline Venetz C, 2011 & sPESI & $\begin{array}{l}134 / 5715 \\
(2.7 \%)\end{array}$ & $\begin{array}{l}1236 / 9816 \\
(13.1 \%)\end{array}$ & Not mentioned & Not mentioned & $\begin{array}{l}69 / 5715 \\
(1.2 \%)\end{array}$ & $\begin{array}{l}275 / 9816 \\
(2.8 \%)\end{array}$ & 0.902 & 0.394 & N/A & N/A & 0.799 & 0.372 \\
\hline Hariharan P, 2011 & PESI & Not mentioned & Not mentioned & Not mentioned & Not mentioned & 9/109 (8.3\%) & 54/136 (39.7\%) & N/A & N/A & N/A & N/A & 0.857 & 0.549 \\
\hline Jiménez D, 2011 & PESI & Not mentioned & Not mentioned & 1/199 (0.5\%) & $36 / 392(9.2 \%)$ & Not mentioned & Not mentioned & N/A & N/A & 0.973 & 0.357 & N/A & N/A \\
\hline $\begin{array}{l}\text { Singanayagam A, } \\
2011\end{array}$ & PESI & $1 / 197(0.5 \%)$ & $22 / 214(10.3 \%)$ & Not mentioned & Not mentioned & Not mentioned & Not mentioned & 0.957 & 0.505 & N/A & N/A & N/A & N/A \\
\hline $\begin{array}{l}\text { Jiménez D, } 2010 \\
\text { (Derivation Cohort) }\end{array}$ & PESI & $89 / 4235$ (2.1\%) & $857 / 6119(14.0 \%)$ & Not mentioned & Not mentioned & Not mentioned & Not mentioned & 0.906 & 0.411 & N/A & N/A & N/A & N/A \\
\hline $\begin{array}{l}\text { Jiménez D, } 2010 \\
\text { (Derivation Cohort) }\end{array}$ & sPESI & $3 / 305(1.0 \%)$ & 75/690 (10.9\%) & Not mentioned & Not mentioned & Not mentioned & Not mentioned & 0.962 & 0.329 & N/A & N/A & N/A & N/A \\
\hline $\begin{array}{l}\text { Jiménez D, } 2010 \\
\text { (RIETE Cohort) }\end{array}$ & sPESI & 28/2565 (1.1\%) & $404 / 4541$ (8.9\%) & Not mentioned & Not mentioned & Not mentioned & Not mentioned & 0.935 & 0.380 & N/A & N/A & N/A & N/A \\
\hline Sam A, 2011 & PESI & 16/431 (3.7\%) & 103/775 (13.3\%) & Not mentioned & Not mentioned & Not mentioned & Not mentioned & 0.866 & 0.382 & N/A & N/A & N/A & N/A \\
\hline Sam A, 2011 & sPESI & $6 / 369(1.6 \%)$ & $113 / 837(13.5 \%)$ & Not mentioned & Not mentioned & $8 / 369(2.2 \%)$ & $34 / 837(4.1 \%)$ & 0.950 & 0.334 & N/A & N/A & 0.810 & 0.310 \\
\hline Chan CM, 2010 & PESI & 0/106 (0.0\%) & 9/196 (4.6\%) & Not mentioned & Not mentioned & Not mentioned & Not mentioned & 1.000 & 0.362 & N/A & N/A & N/A & N/A \\
\hline $\begin{array}{l}\text { Singanayagam A, } \\
2010\end{array}$ & PESI & $6 / 288(2.1 \%)$ & $38 / 297(12.8 \%)$ & Not mentioned & Not mentioned & Not mentioned & Not mentioned & 0.864 & 0.521 & N/A & N/A & N/A & N/A \\
\hline Moores L, 2010 & PESI & $2 / 191(1.0 \%)$ & $55 / 376(14.6 \%)$ & 1/191 (1.5\%) & $33 / 376(8.8 \%)$ & Not mentioned & Not mentioned & 0.965 & 0.371 & 0.971 & 0.356 & N/A & N/A \\
\hline Nordenholz K, 2011 & PESI & $0 / 91(0.0 \%)$ & $5 / 77(6.5 \%)$ & Not mentioned & Not mentioned & Not mentioned & Not mentioned & 1.000 & 0.588 & N/A & N/A & N/A & N/A \\
\hline Choi WH, 2009 & PESI & $3 / 39(7.7 \%)$ & $13 / 51(25.5 \%)$ & Not mentioned & Not mentioned & Not mentioned & Not mentioned & 0.813 & 0.486 & N/A & N/A & N/A & N/A \\
\hline Donzé J, 2008 & PESI & $2 / 186(1.1 \%)$ & 19/171 (11.1\%) & Not mentioned & Not mentioned & Not mentioned & Not mentioned & 0.905 & 0.548 & N/A & N/A & N/A & N/A \\
\hline Jiménez D, 2008 & PESI & 0/100 (0.0\%) & $12 / 218(5.5 \%)$ & Not mentioned & Not mentioned & Not mentioned & Not mentioned & 1.000 & 0.327 & N/A & N/A & N/A & N/A \\
\hline $\begin{array}{l}\text { Jiménez D, } 2007 \\
\text { (Validation Cohort) }\end{array}$ & PESI & $2 / 216(0.9 \%)$ & 41/383 (10.7\%) & Not mentioned & Not mentioned & 6/216 (2.8\%) & 26/383 (6.8\%) & 0.953 & 0.385 & N/A & N/A & 0.813 & 0.370 \\
\hline Aujesky D, 2007 & PESI & $5 / 426(1.2 \%)$ & $53 / 573(9.2 \%)$ & $3 / 426(0.7 \%)$ & 18/573 (3.1\%) & Not mentioned & Not mentioned & 0.914 & 0.447 & 0.857 & 0.433 & N/A & N/A \\
\hline
\end{tabular}


was $0.7853 \pm 0.0058$, and the overall accuracy $\left(Q^{*}\right)$ was $0.7231 \pm 0.0050$. While in the PESI subgroup, the pooled sensitivity was 0.900 ( $95 \% \mathrm{CI}: 0.889$ to 0.911 ), the pooled specificity was 0.411 ( $95 \%$ CI: 0.407 to 0.415 ), the SROC curve is shown in Figure 7B, the overall weighted AUC was $0.7856 \pm 0.0075$, and the overall accuracy $\left(Q^{*}\right)$ was $0.7234 \pm 0.0065$; in the sPESI subgroup, the pooled sensitivity was 0.919 ( $95 \% \mathrm{CI}: 0.907$ to 0.930 ), the pooled specificity was 0.382 ( $95 \% \mathrm{CI}: 0.376$ to 0.388 ), the SROC curve is shown in Figure $7 \mathrm{C}$, the overall weighted AUC was $0.7920 \pm 0.0117$, and the overall accuracy $\left(Q^{*}\right)$ was $0.7289 \pm 0.0101$.
For the outcome of PE-related mortality, the pooled sensitivity was 0.953 ( $95 \% \mathrm{CI}: 0.913$ to 0.978 ). The pooled specificity was 0.374 ( $95 \%$ CI: 0.360 to 0.388 ). The SROC curve is shown in Figure 7D. The overall weighted AUC was $0.8218 \pm 0.0349$, and the overall accuracy $\left(Q^{*}\right)$ was $0.7552 \pm 0.0314$. While in the PESI subgroup, the pooled sensitivity was 0.949 ( $95 \%$ CI: 0.892 to 0.981 ), the pooled specificity was 0.382 ( $95 \%$ CI: 0.362 to 0.401 ), the SROC curve is shown in Figure 7E, the overall weighted AUC was $0.8158 \pm 0.0451$, and the overall accuracy $\left(Q^{*}\right)$ was $0.7498 \pm 0.0403$; in the sPESI subgroup, the pooled sensitivity was 0.960 ( $95 \% \mathrm{CI}: 0.888$ to 0.992 ), the pooled

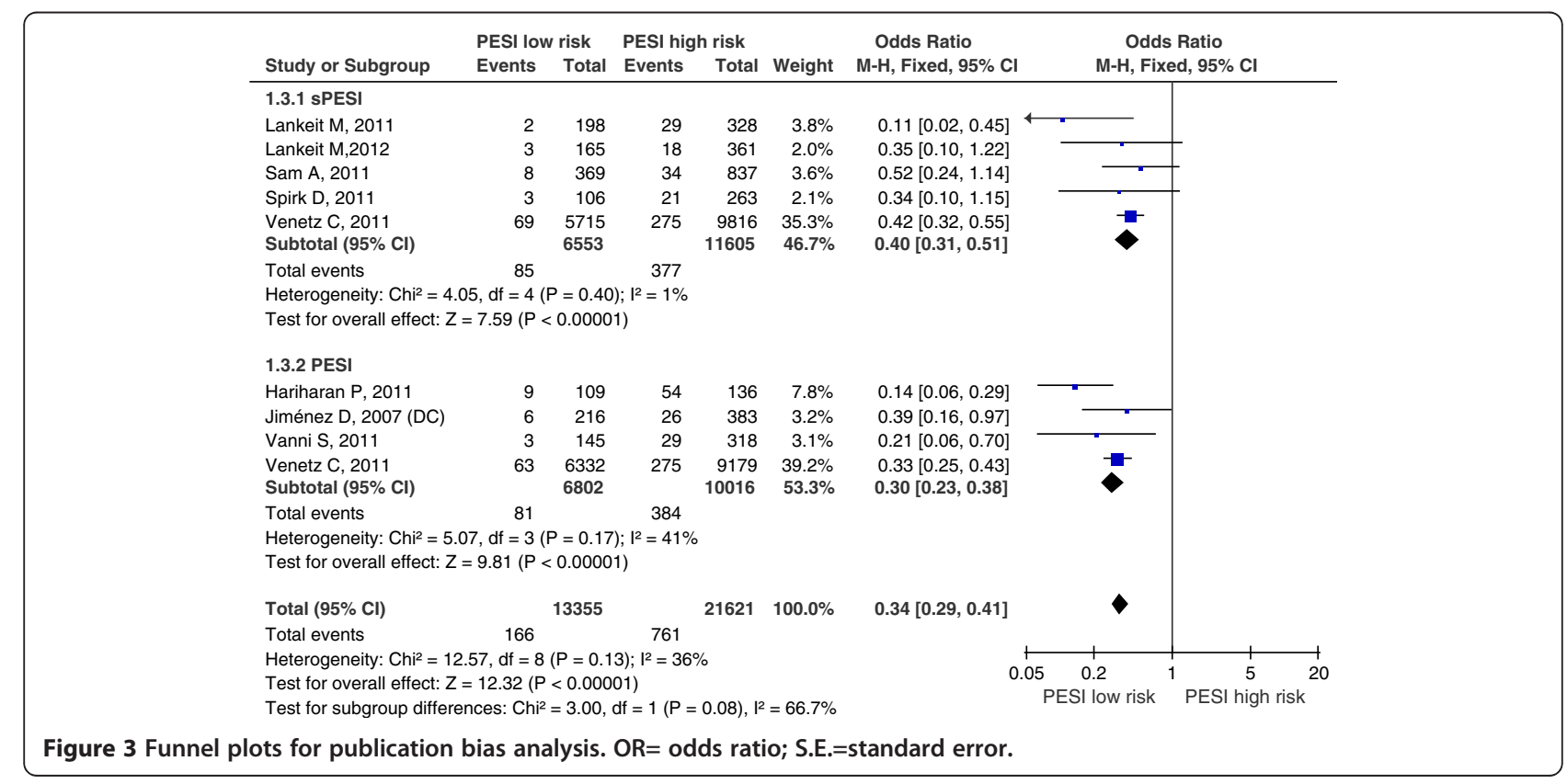


specificity was 0.365 ( $95 \%$ CI: 0.345 to 0.386 ), the SROC curve is shown in Figure 7F, the overall weighted AUC was $0.8317 \pm 0.0547$, and the overall accuracy $\left(Q^{*}\right)$ was $0.7642 \pm 0.0501$.

For the outcome of serious adverse events, the pooled sensitivity was 0.821 ( $95 \% \mathrm{CI}: 0.795$ to 0.845$)$. The pooled specificity was 0.389 (95\% CI: 0.384 to 0.394 ). The SROC curve is shown in Figure 7G. The overall weighted AUC was $0.6809 \pm 0.0208$, and the overall accuracy $\left(Q^{*}\right)$ was $0.6378 \pm 0.0164$. While in the PESI subgroup, the pooled sensitivity was 0.826 (95\% CI: 0.788 to 0.859$)$, the pooled specificity was 0.415 (95\% CI: 0.407 to 0.422 ), the SROC curve is shown in Figure $7 \mathrm{H}$, the overall weighted AUC was $0.7100 \pm 0.0314$, and the overall accuracy $\left(Q^{*}\right)$ was $0.6609 \pm 0.0252$; in the sPESI subgroup, the pooled sensitivity was 0.816 (95\% CI: 0.778 to 0.850$)$, the pooled specificity was $0.366(95 \%$ CI: 0.358 to 0.373$)$, the SROC curve is shown in Figure 7I, the overall weighted AUC was $0.6454 \pm 0.0197$, and the overall accuracy $\left(\mathrm{Q}^{*}\right)$ was $0.6101 \pm 0.0152$.

\section{Discussion}

Some meta-analysis and systematic reviews have provided sufficient evidence that the prognostic outcomes of APE can be affected by haemodynamic status, right ventricular dysfunction, myocardial injury, and other clinical features [37-41]. PESI was designed based on some clinical features. Our meta-analysis showed highrisk PESI is a risk factor for all-cause death, PE-related death, and serious adverse events. Pooled sensitivity, specificity, and SROC were used for predicting PE prognosis. An AUC value of 0.5 indicates that the test has no discriminatory ability, whereas an AUC value of 1.0 indicates perfect capability [42]. While to demonstrate excellent accuracy, the AUC should be in the region of 0.97 or above; an AUC of 0.93 to 0.96 is very good; 0.75 to 0.92 is good; but an AUC less than 0.75 has obvious deficiencies in its accuracy [43]. Our meta-analysis showed the overall weighted AUC for all-cause mortality was 0.78 ( $95 \%$ CI: 0.77 to 0.80 ); and AUC for PE-related mortality was 0.82 (95\% CI: 0.75 to 0.89 ). PESI has good accuracy for predicting PE prognosis. The main reason is that the PESI was derived form multi-center large sample studies with appropriate research methods. In the PESI study, 15,531 discharged patients with PE treated at 186 hospitals were included, on the basis of the $\beta$-coefficients of the stepwise logistic regression model, PESI with a point score was generated [9].

Our meta-analysis showed in sPESI subgroup, the OR of all-cause mortality, PE-related mortality, and serious adverse events was similar to that in PESI subgroup. Most important, in sPESI subgroup, the AUC for predicting all-cause mortality, PE-related mortality, and serious adverse events was also similar to that in PESI subgroup. It has the same accuracy between PESI and sPESI. The sPESI is also derived form logistic regression analysis [10]. In the derivation data set, univariate logistic regression of the original 11 PESI variables led to the removal of variables that did not reach statistical significance and subsequently produced the simplified PESI that contained the variables of cancer, chronic cardiopulmonary 

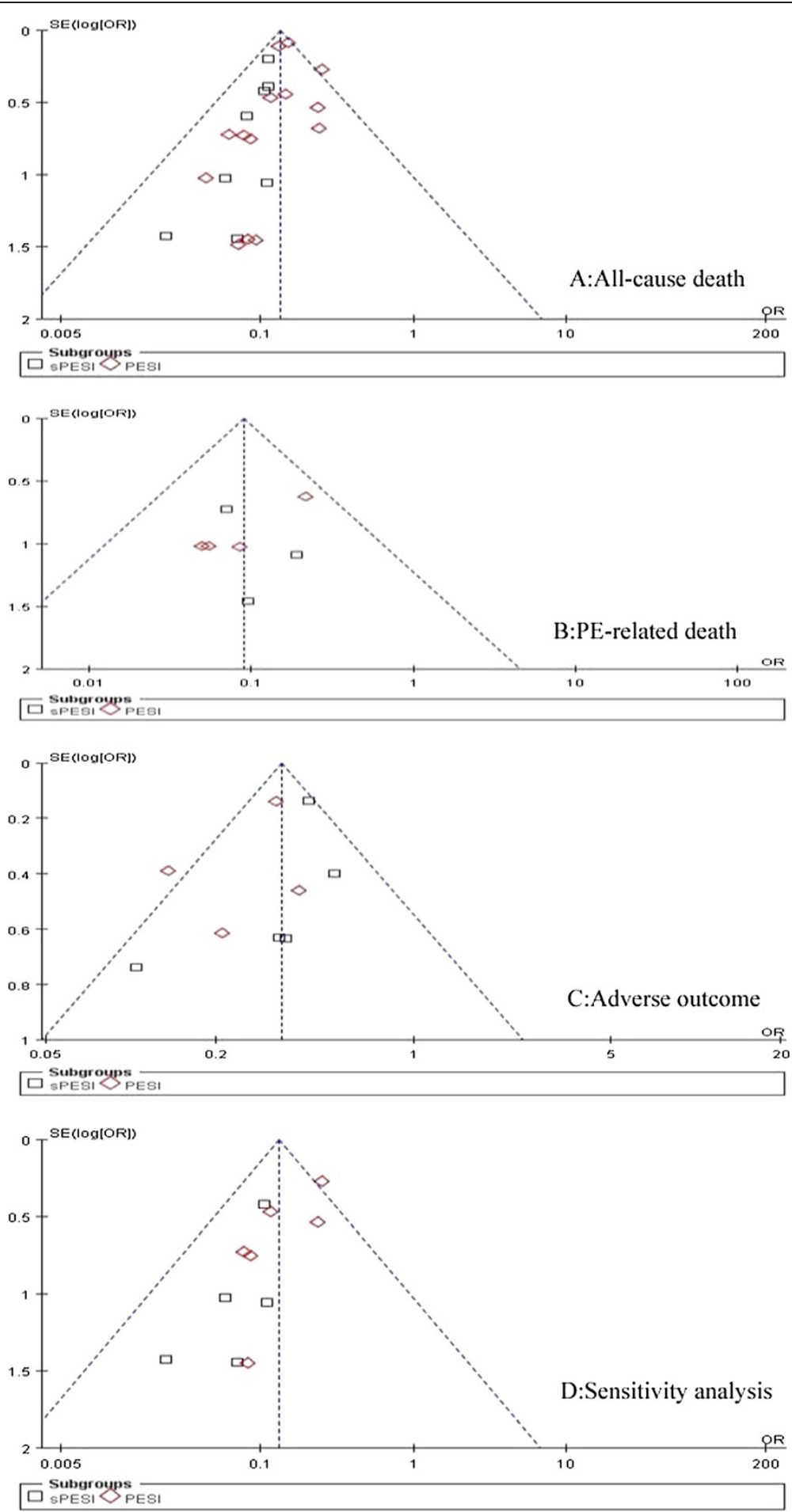

Figure 5 Meta-analysis of serious adverse events in PE with PESI low risk versus PESI high risk. Serious adverse events were defined as any of the following: nonfatal recurrent $P E$, nonfatal recurrent DVT, nonfatal bleeding, or delayed homodynamic instability. 


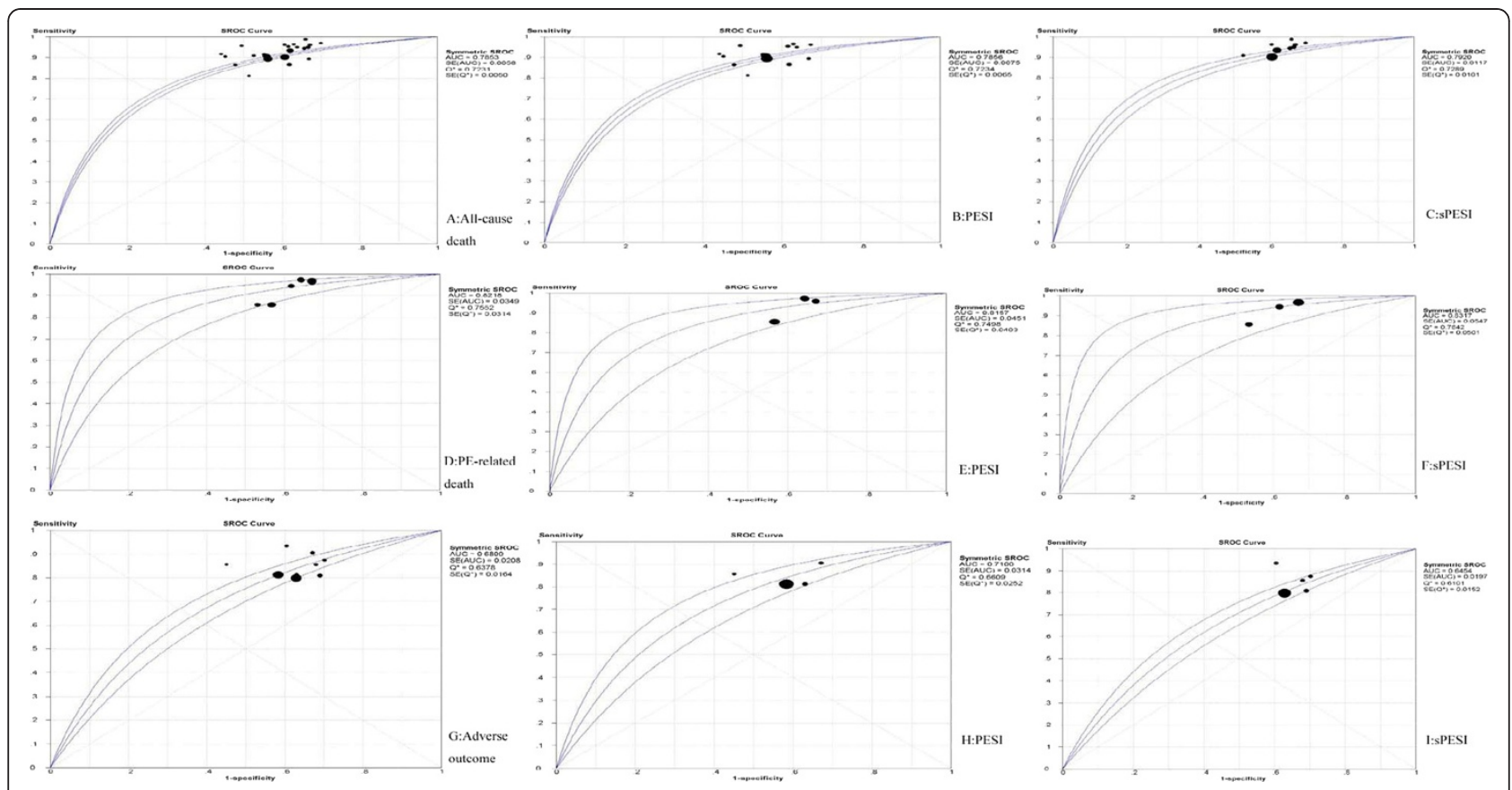

Figure 6 Sensitivity analysis of all-cause mortality (primary outcome) by removal the retrospective studies.

disease, heart rate, systolic blood pressure, and oxyhemoglobin saturation levels. 11 variables in PESI reduced to 6 variables in SPESI. The sPESI does not decrease prognostic accuracy compared with the original PESI, but sPESI is easier to use.

In general, the performance of prognostic models should be assessed in two ways: its discrimination and calibration aspects. Discrimination is the ability of the model to correctly separate the subjects into different groups. Calibration is the degree of correspondence between the estimated probability produced by the model

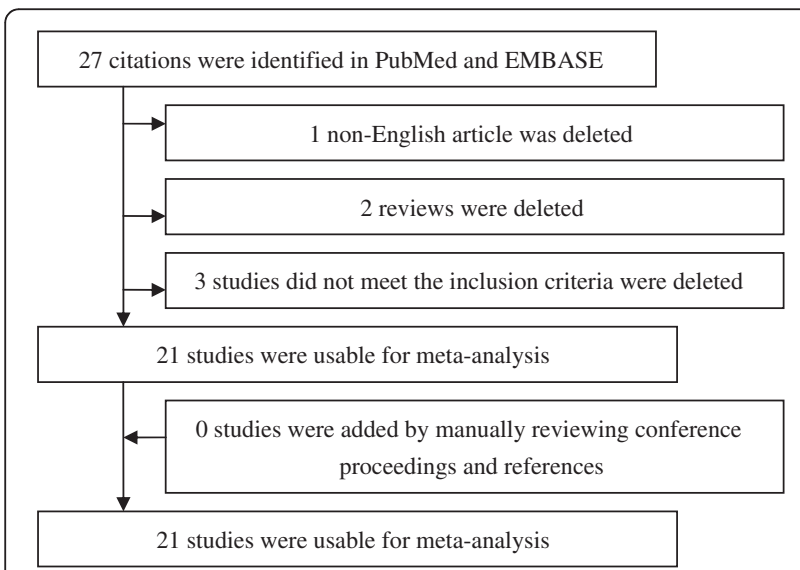

Figure 7 SROC plots for combined sensitivity and (1-specificity) of the included studies. and the actual observed probability [6]. Discrimination is also called predicting accuracy, which can be assessed by sensitivity (true positive rate), and specificity (true negative rate), ROC, and AUC. Our meta-analysis has already proved the PESI has good accuracy for predicting APE outcomes. The calibration is also assessed by the Hosmer-Lemeshow test [44]. But the data of the calibration was not found in the included studies. So we can't calculate the calibration for predicting prognosis in this meta-analysis. Some prospective studies for accessing PESI predicting calibration can be recommended.

Except we can't calculated the calibration for predicting prognosis in this meta-analysis, there are some other limitations. First, Lankeit $M$ maybe used same participants in two included studies [17] and [19], as well as Singanayagam A's studies [26,29], and Jiménez D's studies $[34,35]$. Repeated include will increase the weight of the studies, and maybe result bias. Second, different PE haemodynamic status of patients included in our metaanalysis, some included haemodynamic stable patients, some included haemodynamic stable and unstable patients. But this bias is small, because evaluation of hemodynamic status is included in the PESI and sPESI. Third, the meta-analysis can't test the prognostic accuracy against other clinical score such as the Geneva prognostic score. Finally, most of the included studies were came from Europe and North America, only a study came from Asia, no study came from Africa, South America and Oceania. Some high-quality studies should 
be performed in these areas before PESI used worldwide.

\section{Conclusions}

Our meta-analysis identified PESI has discriminative power to predict the short-term death and adverse outcome events in patients with acute pulmonary embolism; the PESI and the sPESI have similar accuracy, while sPESI is easier to use. However, the calibration for predicting prognosis can't be calculated from this meta-analysis, some prospective studies for accessing PESI predicting calibration can be recommended.

\section{Additional files}

Additional file 1: Pulmonary Embolism Severity Index (PESI), Simplified PESI (sPESI).

Additional file 2: Newcastle - Ottawa Quality Assessment Scale.

\section{Competing interests}

The authors declare that they have no competing interests.

\section{Authors' contributions}

XYZ and HLC reviewed the full text articles, extracted data and wrote the initial draft of the manuscript. SQB conducted the literature search, reviewed titles, abstracts and full text articles and contributed to the extraction of data. $\mathrm{HLC}$ reviewed titles and abstracts and provided critical revision of the manuscript. SSN and HLC contributed to the critical revision of the manuscript. All authors read and approved the final manuscript.

\section{Author details}

'Department of Respiratory Diseases, the Affiliated Hospital of Nantong University, Xi Si Road 20\#, Nantong City, Jiangsu Province 226001, People's Republic of China. ${ }^{2}$ Nantong University, Qi Xiu Road 19\#, Nantong City, Jiangsu Province 226001, People's Republic of China.

Received: 11 July 2012 Accepted: 15 October 2012

Published: 4 December 2012

\section{References}

1. Stein PD, Beemath A, Olson RE: Trends in the incidence of pulmonary embolism and deep venous thrombosis in hospitalized patients. Am J Cardiol 2005, 95(12):1525-1526.

2. Kroeger $\mathrm{K}$, Moerchel $\mathrm{C}$, Moysidis T, et al: Incidence rate of pulmonary embolism in Germany: data from the federal statistical office. J Thromb Thrombolysis 2010, 29(3):349-353.

3. Yuanhua $Y$, Lirong $L$, Zhenguo Z, et al: Pulmonary Embolism Incidence and Fatality Trends in Chinese Hospitals from 1997 to 2008: A Multicenter Registration Study. PLoS One 2011, 6(11):e26861.

4. Choi Won-II, Lee Mi-Young, Doyeun O, et al: Estimated Incidence of Acute Pulmonary Embolism in a Korean Hospital. Clin Appl Thromb Hemost 2011, 17(3):297-301

5. Torbicki A, Perrier A, Konstantinides S, et al: Guidelines on the diagnosis and management of acute pulmonary embolism: the Task Force for the Diagnosis and Management of Acute Pulmonary Embolism of the European Society of Cardiology (ESC). Eur Heart J 2008, 29(18):2276-2315.

6. Torbicki A, Galie N, Covezzoli A, et al: Right heart thrombi in pulmonary embolism - Results from the International Cooperative Pulmonary Embolism Registry. J Am Coll Cardiol 2003, 41(12):2245-2251.

7. Silvy L, Patrick $M$, Herve $D$, et al: Clinical predictors for fatal pulmonary embolism in 15,520 patients with venous thromboembolism - Findings from the Registro Informatizado de la Enfermedad TromboEmbolica venosa (RIETE) registry. Circulation 2008, 117(13):1711-1716.

8. Pollack Charles V, Schreiber D, Goldhaber Samuel Z, et al: Clinical Characteristics, Management, and Outcomes of Patients Diagnosed With
Acute Pulmonary Embolism in the Emergency Department Initial Report of EMPEROR (Multicenter Emergency Medicine Pulmonary Embolism in the Real World Registry). J Am Coll Cardiol 2011, 57(6):700-706.

9. Aujesky D, Obrosky DS, Stone RA, et al: Derivation and validation of a prognostic model for pulmonary embolism. Am J Respir Crit Care Med 2005, 172(8):1041-1046.

10. Jiménez D, Aujesky D, Moores L, Gómez V, Lobo JL, Uresandi F, Otero R, Monreal M, Muriel A, Yusen RD: RIETE Investigators: Simplification of the pulmonary embolism severity index for prognostication in patients with acute symptomatic pulmonary embolism. Arch Intern Med 2010, 9;170 (15):1383-1389.

11. GA Wells, B Shea, D O'Connell, J Peterson, V Welch, M Losos, P Tugwell: The Newcastle-Ottawa Scale (NOS) for assessing the quality of nonrandomized studies in meta-analysis. Ottawa Health Research Institute. Available from: URL: http://www.ohri.ca/programs/clinical_epidemiology/ oxford.asp (assessed 10 June 2012).

12. Higgins JP, Thompson SG: Quantifying heterogeneity in a meta-analysis. Stat Med 2002, 21(11):1539-1558.

13. DerSimonian R, Laird N: Meta-analysis in clinical trials. Control Clin Trials 1986, 7:177-188

14. Moses LE, Shapiro D, Littenberg B: Combining independent studies of a diagnostic test into a summary ROC curve: data-analytic approaches and some additional considerations. Stat Med 1993, 12:1293-1316.

15. Walter SD: Properties of the summary receiver operating characteristic (sROC) curve for diagnostic test data. Stat Med 2002, 21:1237-1256.

16. Zamora J, Abraira V, Muriel A, Khan K, Coomarasamy A: Meta-Disc: a software for meta analysis of test accuracy data. BMC Med Res Methodol 2006, 6:31-47.

17. Lankeit M, Jiménez D, Kostrubiec M, Dellas $C$, Hasenfuss G, Pruszczyk $P$, Konstantinides S: Predictive value of the high-sensitivity troponin $\mathrm{T}$ assay and the simplified Pulmonary Embolism Severity Index in hemodynamically stable patients with acute pulmonary embolism: a prospective validation study. Circulation 2011 13;124(24):2716-2724.

18. Sánchez D, De Miguel J, Sam A, Wagner C, Zamarro C, Nieto R, García L, Aujesky D, Yusen RD, Jiménez D: The effects of cause of death classification on prognostic assessment of patients with pulmonary embolism. J Thromb Haemost 2011, 9(11):2201-2207.

19. Lankeit M, Gómez V, Wagner C, Aujesky D, Recio M, Briongos S, Moores LK, Yusen RD, Konstantinides S, Jiménez D, Instituto Ramón y Cajal de Investigación Sanitaria Pulmonary Embolism Study Group: A strategy combining imaging and laboratory biomarkers in comparison with a simplified clinical score for risk stratification of patients with acute pulmonary embolism. Chest 2012, 141(4):916-922.

20. Righini M, Roy PM, Meyer G, Verschuren F, Aujesky D, Le Gal G: The Simplified Pulmonary Embolism Severity Index (PESI): validation of a clinical prognostic model for pulmonary embolism. J Thromb Haemost 2011, 9(10):2115-2117.

21. Spirk D, Aujesky D, Husmann M, Hayoz D, Baldi T, Frauchiger B, Banyai M, Baumgartner I, Kucher N: Cardiac troponin testing and the simplified Pulmonary Embolism Severity Index. The SWIss Venous ThromboEmbolism Registry (SWIVTER). Thromb Haemost 2011, 106(5):978-984.

22. Vanni S, Nazerian P, Pepe G, Baioni M, Risso M, Grifoni G, Viviani G, Grifoni S: Comparison of two prognostic models for acute pulmonary embolism: clinical vs. right ventricular dysfunction-guided approach. J Thromb Haemost 2011, 9(10):1916-1923.

23. Venetz $C$, Jiménez $D$, Mean $M$, Aujesky D: A comparison of the original and simplified Pulmonary Embolism Severity Index. Thromb Haemost 2011, 106(3):423-428.

24. Hariharan P, Takayesu JK, Kabrhel C: Association between the Pulmonary Embolism Severity Index (PESI) and short-term clinical deterioration. Thromb Haemost 2011, 105(4):706-711.

25. Jiménez D, Aujesky D, Moores L, Gómez V, Martí D, Briongos S, Monreal M, Barrios V, Konstantinides S, Yusen RD: Combinations of prognostic tools for identification of high-risk normotensive patients with acute symptomatic pulmonary embolism. Thorax 2011, 66(1):75-81.

26. Singanayagam A, Scally C, Al-Khairalla MZ, Leitch L, Hill LE, Chalmers JD, Hill AT: Are biomarkers additive to pulmonary embolism severity index for severity assessment in normotensive patients with acute pulmonary embolism? QJM 2011, 104(2):125-131. 
27. Sam A, Sánchez D, Gómez V, Wagner C, Kopecna D, Zamarro C, Moores L, Aujesky D, Yusen R, Jiménez Castro D: The shock index and the simplified PESI for identification of low-risk patients with acute pulmonary embolism. Eur Respir J 2011, 37(4):762-766.

28. Chan CM, Woods C, Shorr AF: The validation and reproducibility of the pulmonary embolism severity index. J Thromb Haemost 2010, 8(7):1509-1514.

29. Singanayagam A, Chalmers JD, Scally C, Akram AR, Al-Khairalla MZ, Leitch L, Hill LE, Hill AT: Right ventricular dilation on CT pulmonary angiogram independently predicts mortality in pulmonary embolism. Respir Med 2010, 104(7):1057-1062.

30. Moores L, Aujesky D, Jiménez D, Díaz G, Gómez V, Martí D, Briongos S, Yusen R: Pulmonary Embolism Severity Index and troponin testing for the selection of low-risk patients with acute symptomatic pulmonary embolism. J Thromb Haemost 2010, 8(3):517-522.

31. Nordenholz K, Ryan J, Atwood B, Heard K: Pulmonary embolism risk stratification: pulse oximetry and pulmonary embolism severity index. J Emerg Med 2011, 40(1):95-102.

32. Choi WH, Kwon SU, Jwa YJ, Kim JA, Choi YH, Chang JH, Jung H, Doh JH, Namgung J, Lee SY, Lee WR: The pulmonary embolism severity index in predicting the prognosis of patients with pulmonary embolism. Korean J Intern Med 2009, 24(2):123-127.

33. Donzé J, Le Gal G, Fine MJ, Roy PM, Sanchez O, Verschuren F, Cornuz J, Meyer G, Perrier A, Righini M, Aujesky D: Prospective validation of the Pulmonary Embolism Severity Index. A clinical prognostic model for pulmonary embolism. Thromb Haemost 2008, 100(5):943-948.

34. Jiménez D, Díaz G, Molina J, Martí D, Del Rey J, García-Rull S, Escobar C, Vidal R, Sueiro A, Yusen RD: Troponin I and risk stratification of patients with acute nonmassive pulmonary embolism. Eur Respir J 2008, 31(4):847-853.

35. Jiménez D, Yusen RD, Otero R, Uresandi F, Nauffal D, Laserna E, Conget F, Oribe M, Cabezudo MA, Díaz G: Prognostic models for selecting patients with acute pulmonary embolism for initial outpatient therapy. Chest 2007, 132(1):24-30.

36. Aujesky D, Perrier A, Roy PM, Stone RA, Cornuz J, Meyer G, Obrosky DS, Fine MJ: Validation of a clinical prognostic model to identify low-risk patients with pulmonary embolism. J Intern Med 2007, 261(6):597-604.

37. Coutance G, Cauderlier E, Ehtisham J, et al: The prognostic value of markers of right ventricular dysfunction in pulmonary embolism: a meta-analysis. CRITICAL CARE 2011, 2(15):R103.

38. Klok Frederikus A, Mos Inge CM, Huisman Menno V: Brain-type natriuretic peptide levels in the prediction of adverse outcome in patients with pulmonary embolism - A systematic review and meta-analysis. Am J Respir Crit Care Med 2008, 178(4):425-430.

39. Lega J-C, Lacasse $Y$, Lakhal L: Natriuretic peptides and troponins in pulmonary embolism: a meta-analysis. Thorax 2009, 64(10):869-875.

40. Becattini C, Vedovati Maria C, Agnelli G: Prognostic value of troponins in acute pulmonary embolism - A meta-analysis. Circulation 2007, 116(4):427-433.

41. West J, Goodacre S, Sampson F: The value of clinical features in the diagnosis of acute pulmonary embolism: systematic review and meta-analysis. QJM-An International Journal of Medicine 2007, 100(12):763-769.

42. Swets JA: Measuring the accuracy of diagnostic systems. Science 1988 240:1285-1293.

43. Jones CM, Athanasiou T: Summary receiver operating characteristic curve analysis techniques in the evaluation of diagnostic tests. Ann Thorac Surg 2005, 79:16-20.

44. Kramer Andrew A, Zimmerman Jack E: Assessing the calibration of mortality benchmarks in critical care: The Hosmer-Lemeshow test revisited. Crit Care Med 2007, 35(9):2052-2056.

\section{doi:10.1186/1465-9921-13-111}

Cite this article as: Zhou et al:: The prognostic value of pulmonary embolism severity index in acute pulmonary embolism: a meta-analysis. Respiratory Research 2012 13:111.

\section{Submit your next manuscript to BioMed Central and take full advantage of:}

- Convenient online submission

- Thorough peer review

- No space constraints or color figure charges

- Immediate publication on acceptance

- Inclusion in PubMed, CAS, Scopus and Google Scholar

- Research which is freely available for redistribution

Submit your manuscript at www.biomedcentral.com/submit
C BioMed Central 